\title{
Facial skin pores: a multiethnic study
}

This article was published in the following Dove Press journal:

Clinical, Cosmetic and Investigational Dermatology

16 February 2015

Number of times this article has been viewed

\section{Frederic Flament ${ }^{1}$ \\ Ghislain Francois' \\ Huixia Qiu² \\ Chengda $\mathrm{Ye}^{2}$ \\ Tomoo Hanaya ${ }^{3}$ \\ Dominique Batisse ${ }^{3}$ \\ Suzy Cointereau-Chardon' \\ Mirela Donato Gianeti \\ Seixas ${ }^{4}$ \\ Susi Elaine Dal Belo ${ }^{4}$ \\ Roland Bazin ${ }^{5}$ \\ 'Department of Applied Research and Development, L'Oreal Research and Innovation, Paris, France; ${ }^{2}$ Department of Applied Research and Development, L'Oreal Research and Innovation, Shanghai, People's Republic of China; ${ }^{3}$ Department of Applied Research and Development, L'Oreal Research and Innovation, Tokyo, Japan; ${ }^{4}$ Department of Applied Research and Development, L'Oreal Research and Innovation, Rio de Janeiro, Brazil; ${ }^{5}$ RB Consult, Bievres, France}

Correspondence: Frederic Flament Department of Applied Research and Development, L'Oreal Research and Innovation, 188, Rue Paul Hochart, 94550 Chevilly-Larue, Paris, France

Email fflament@rd.loreal.com
Abstract: Skin pores (SP), as they are called by laymen, are common and benign features mostly located on the face (nose, cheeks, etc) that generate many aesthetic concerns or complaints. Despite the prevalence of skin pores, related literature is scarce. With the aim of describing the prevalence of skin pores and anatomic features among ethnic groups, a dermatoscopic instrument, using polarized lighting, coupled to a digital camera recorded the major features of skin pores (size, density, coverage) on the cheeks of 2,585 women in different countries and continents. A detection threshold of $250 \mu \mathrm{m}$, correlated to clinical scorings by experts, was input into a specific software to further allow for automatic counting of the SP density $\left(\mathrm{N} / \mathrm{cm}^{2}\right)$ and determination of their respective sizes in $\mathrm{mm}^{2}$. Integrating both criteria also led to establishing the relative part of the skin surface (as a percentage) that is actually covered by SP on cheeks. The results showed that the values of respective sizes, densities, and skin coverage: 1) were recorded in all studied subjects; 2) varied greatly with ethnicity; 3 ) plateaued with age in most cases; and 4) globally reflected self-assessment by subjects, in particular those who self-declare having "enlarged pores" like Brazilian women. Inversely, Chinese women were clearly distinct from other ethnicities in having very low density and sizes. Analyzing the present results suggests that facial skin pore's morphology as perceived by human eye less result from functional criteria of associated appendages such as sebaceous glands. To what extent skin pores may be viewed as additional criteria of a photo-altered skin is an issue to be further addressed.

Keywords: aging, clinical evaluation, ethnicity, imperfections, oily skin

\section{Introduction}

Dermatologists, cosmeticians, and aestheticians often face challenging questions from their patients or customers about the presence of large or enlarged skin pores (SP) and the practical possibilities to alleviate such undesired aspects. SP nonetheless appear to be a genuine clinical entity and a concern of many people. ${ }^{1}$ Although benign, they generate aesthetic complaints and are perceived by many as markers of aging skin. These facial skin signs remain the objects of a few researches, they are rarely mentioned in textbooks, and their causative factor/factors remains/remain obscure. ${ }^{1}$

The word "pore", although inappropriate, is a term used by millions of laypeople worldwide. Physiologically speaking, pores are defined as apertures from the surface of a tegument (animal, vegetal) that ensure the input or output of gases or fluids. With regard to skin, such orifices only comprise the tiny ostia from either pilosebaceous follicles or eccrine sweat glands. The latter are rather evenly distributed along the human face (scalp not included) with a comparable density $\left(200-300 / \mathrm{cm}^{2}\right)$; are of different sizes, ie, $40-80 \mu \mathrm{m}$ and approximately 5-10 $\mu \mathrm{m}$ in diameter, respectively; ${ }^{2,3}$ 
and are invisible to the naked eye as they are of a size below normal visual acuity. As a matter of fact, dermatologists need magnifying glasses in detecting facial closed comedones in acne. According to previous works, ${ }^{4-6} \mathrm{SP}$ are of much larger dimensions, ie, they are visible to the naked eye, ranging from 250 to $500 \mu \mathrm{m}$ in size, leading to surface areas of $0.05-0.2$ $\mathrm{mm}^{2}$ and above, assuming they have a roundish shape. Macroscopically, they appear as tiny cuvettes conferring a "golf ball"-like aspect to the skin microrelief - of variable densities on cheeks (approximately $10-90 / \mathrm{cm}^{2}$ ), and the surface of the SP are often self-claimed to enlarge with age.

SP can hardly be mixed up with the ostia of pilosebaceous or sweat ducts. Comparing the respective sizes of the former and the latter makes it clear that any SP hosts a significant number of pilosebaceous and eccrine sweat ostia. In brief, SP cannot be seen as true "pores" but are instead slight depressions of the skin surface ${ }^{1,7}$ containing many sebaceous and sweat ostia, ie, not physiological cutaneous aperture entities. Equating these SP to pilosebaceous ostia likely explains the assumption that the sebaceous function (or past acne history) is the most probable causative factor. ${ }^{8-10}$

With regard to such uncertainties, the present work attempted to shed some light upon various aspects of these facial features and, as a preliminary step, to explore their prevalence and characteristics (density, size, coverage) in women of various ethnicities and of different ages. A recently available imaging instrument ${ }^{11}$ allowed for the recording of magnified images of the skin surface and subsequent analysis, yielding both the density $\left(\mathrm{N} / \mathrm{cm}^{2}\right)$ and size (in $\mathrm{mm}^{2}$ ) of SP and the relative portion of skin surface (as a percentage) that they cover. This equipment was applied to the cheeks of large cohorts of women from different countries (Brazil, People's Republic of China, France, India, Japan) and of various ages. A special focus was given to the 30\% largest SP in subjects who self-declared having "enlarged" pores. The major findings of these multiethnic surveys are the foci of the present paper.

\section{Materials and methods Subjects}

There were 2,585 women, aged 18-80 years old (the lower and upper limits, respectively, of the included age range), selected and examined in their own cities of residence, as indicated in Table 1. No particular exclusion criteria were used, apart from non-respect of age range and facial skin presenting clinical disorders (acne, melasma, vitiligo, etc) other than age-related signs. All subjects were informed about the objective of the study and signed an informed consent.
Table I Distribution of the 2,585 study subjects and their relative age ranges in the four countries where imaging of skin pores was performed

\begin{tabular}{lll}
\hline Countries (cities) & $\begin{array}{l}\text { Number of } \\
\text { subjects }\end{array}$ & $\begin{array}{l}\text { Age range } \\
\text { (years) }\end{array}$ \\
\hline People's Republic of China (Shanghai) & 395 & $18-80$ \\
France (Paris) & 218 & $18-65$ \\
India (Mumbai, Delhi, Chennai, Kokalta) & 1,194 & $18-70$ \\
Japan (Tokyo) & 778 & $18-80$ \\
\hline
\end{tabular}

Studies were carried out in all countries during their respective winter seasons, between 2009 and 2011, in various locations (cities/countries) as shown in Table 1. In a further step, a subcohort of 840 women, drawn from the 2,585 previous subjects, was created by arbitrarily selecting, in each age class, the $30 \%$ of subjects with the largest SP.

In addition to these 840 women, 120 Brazilian women who self-declared having enlarged pores and oily skin (a rather frequent complaint in Brazil) were recruited by the L'Oreal Research facility in Rio de Janeiro, to extend the size of cohorts and ethnic profiles (Table 2).

Prior to dermatoscopic recordings, the surface of the cheeks under scope were gently wiped off using a bland cotton pad to avoid shine as much as possible.

\section{Instrumental procedures, threshold level, and digital treatments}

These were carried out with the Dermascore ${ }^{\circledR}$ system (Figure 1), a portable dermatoscope with polarized lighting, which allows for a $4 \times$ magnification of the circular surface of the skin (cheeks) under scope (35 $\mathrm{mm}$ in diameter) with a lateral resolution of $25 \mu \mathrm{m}$. A video camera connected to a computer allowed for recording and storage of images of the skin area in a few seconds. These were further analyzed by a specifically designed software for automatically detecting SP at various possible threshold levels, yielding further values of their density and respective size. The successive steps of

Table 2 Distribution of the 960 subjects claiming to have large skin pores and their relative age ranges in five countries, from which the $30 \%$ of subjects with the largest SP arbitrarily selected and analyzed in all age classes

\begin{tabular}{lll}
\hline Countries (cities) & $\begin{array}{l}\text { Number of } \\
\text { subjects }\end{array}$ & $\begin{array}{l}\text { Age range } \\
\text { (years) }\end{array}$ \\
\hline Brazil (Rio de Janeiro) & 120 & $18-60$ \\
People's Republic of China (Shanghai) & 120 & $18-80$ \\
France (Paris) & 70 & $18-65$ \\
India (Mumbai, Delhi, Chennai, Kokalta) & 400 & $18-70$ \\
Japan (Tokyo) & 250 & $18-80$ \\
\hline
\end{tabular}


A

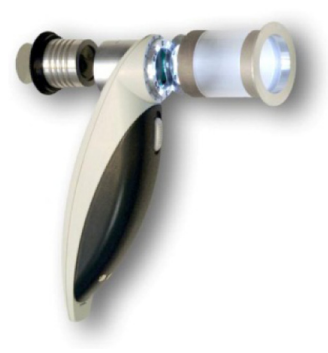

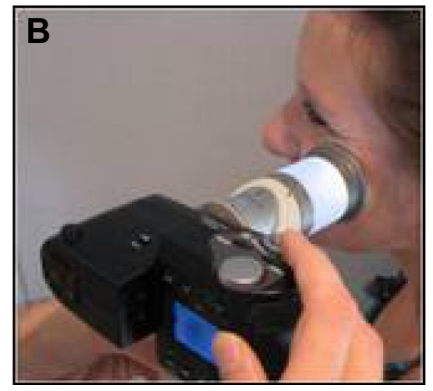

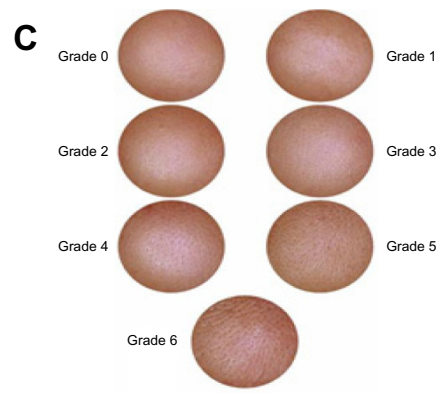

Figure I DermaScore ${ }^{\circledR}$ device.

Notes: (A) DermaScore ${ }^{\circledast}$ device; (B) application onto the cheek; (C) and resulting standardized photographs from different prevalence of skin pores.

instrumental and imaging processes are described below, as previously published. ${ }^{11}$

\section{Threshold determination of sizes of SP}

This procedure ${ }^{11}$ was carried out for selecting the most accurate threshold value to be input into the software, ie, the minimum size value that best fits with average clinical scorings of SP, from 0 (absent) to 6 (extreme presence) (Figure 1C). Previously recorded photographs from the cheeks of 350 women of various ages (150 from France, 200 from the People's Republic of China) were randomly given to a panel of 12 trained experts, under the guidance of standard photographs from the previously edited Skin Atlas, ${ }^{12}$ for attributing a $0-6$ score to each photograph. Evaluations took place in entirely standardized conditions of lighting and position, with experts being seated $1 \mathrm{~m}$ from a high-resolution screen (24 inches, 1,920×1,200 pixels) calibrated with a colorimeter. To ensure the robustness of this process, several pictures were presented twice at random during evaluations. All data were recorded and further averaged for each subject. In a subsequent phase, various thresholds of automatic detection, from $100 \mu \mathrm{m}$ up to 500 $\mu \mathrm{m}$ (as maximum sizes or diameter, assuming a roundish shape) by successive steps of $50 \mu \mathrm{m}$, were provided as input.
Figure 2 illustrates the different patterns obtained on the same picture from different thresholds values.

Correlations between averaged scorings and sizes of SP, for each threshold value, were further carried out using SPSS package software. In both cohorts (Chinese and French), the best coefficients of correlation $(r=0.76$, Caucasian and $r=0.82$, Asian, $P<0.01$ ) were both found at a $250 \mu \mathrm{m}$ threshold (as shown in Figure 3 for the Chinese volunteers), leading to individual pore surfaces ranging from 0.06 to $0.16 \mathrm{~mm}^{2}$. Lower and higher thresholds led to much less significant $r$ values. Such a threshold was therefore systematically used in the multicentric study explained above as it was found acceptable, taking into account inevitable variations in scorings between experts and the limits of precision offered by the software processing.

\section{Influence of skin color upon skin imaging and digital analysis}

The validity of the image analysis obviously depended upon contrasts, a factor that appeared to be only influenced by darker natural skin colors such as those found in some Indian locations. For example, images collected from Indian women in Chennai, where the darkest tones predominate, led to much less reproducible data, requiring rechecks by manual counting
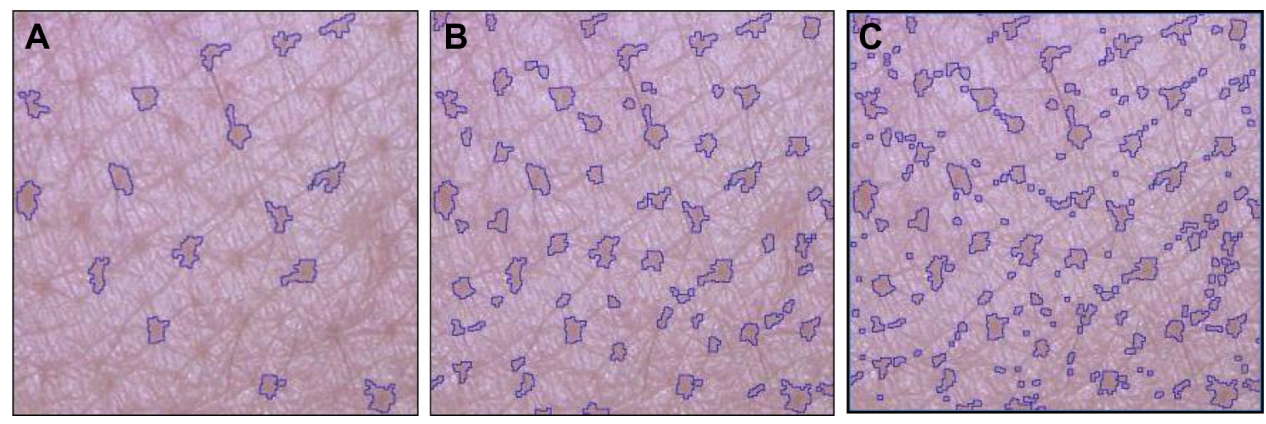

Figure 2 Impact of various input thresholds upon automatic detection of facial skin pores.

Notes: From left to right, high to low thresholds: (A) $500 \mu \mathrm{m}$ threshold; (B) $250 \mu \mathrm{m}$ threshold; (C) $100 \mu \mathrm{m}$ threshold. 


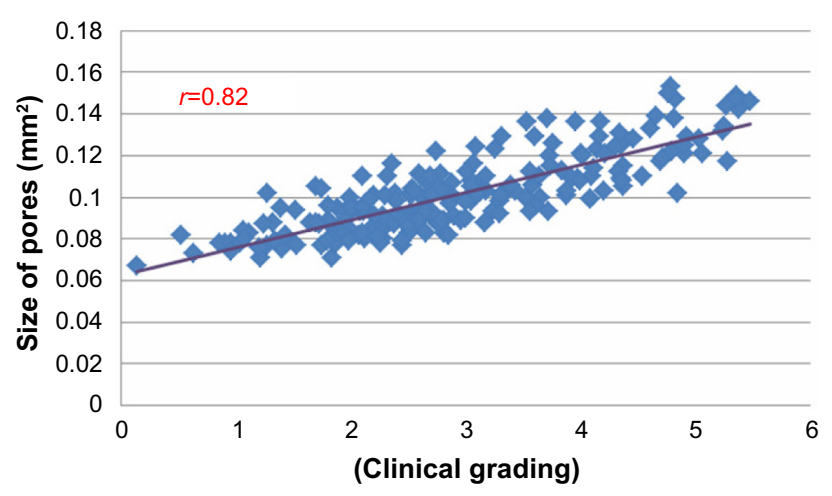

Figure 3 Example of correlation obtained between clinical scorings and skin pore surface in 200 Chinese women, using a $250 \mu \mathrm{m}$ threshold of detection.

and sizing: detections were validated as efficient. Applying this technique to dark-skinned volunteers is a limiting factor that prevented us from extending our recordings to darkskinned African women. Work is currently being undertaken to overcome such restrictive technical issue. With regard to fairer skin tones, Brazilian women included $(70 \%$ of Brazilian panels comprise Fitzpatrick skin types $\leq$ IV), the reproducibility of measurements appeared high (variation coefficient around $3 \%$ ) and was considered as satisfactory.

\section{Statistical analysis}

Analysis of variance with a post hoc pairwise multiple comparisons and range test (Tukey's) were used to compare pore characteristics of all ethnic groups (factor: ethnicity) in each 10-year cluster and to evaluate the age effect (factor: age group). IBM SPSS Statistic version 20 (IBM
Corporation, Armonk, NY, USA) was used to perform all of these analyses.

\section{Results \\ Effect of ethnicity and age upon density of SP}

Figure 4 illustrates changes in the density of SP (mean $\pm 95 \%$ confidence interval) with age among the four ethnic groups, with all 2,585 subjects included. Although densities of SP show high variability among the four ethnic groups studied (ranging from 10 to $80 / \mathrm{cm}^{2}$, an approximate eightfold factor), age appears to have a nonsignificant impact within each group. A slight decline in densities with age is even noticeable among Indian and Caucasian women. Chinese women clearly differ from the three other groups, in that they display very low densities.

\section{Effects of ethnicity and age upon sizes of SP}

Figure 5 illustrates changes in size of SP with age among the four ethnic groups studied, with all 2,585 subjects included. Average sizes of SP show a rather high variability among ethnic groups, ranging from 0.03 to $0.22 \mathrm{~mm}^{2}$, an approximate sevenfold factor. A significant increase in SP size with age is observed among Indian women, with the three other groups showing rather stable values with age, ie, for 18-29 year olds, cluster size is significantly lower and it plateaus from 40 years of age. Again, Chinese women were clearly distinct from the three other groups, in that they displayed very low values.

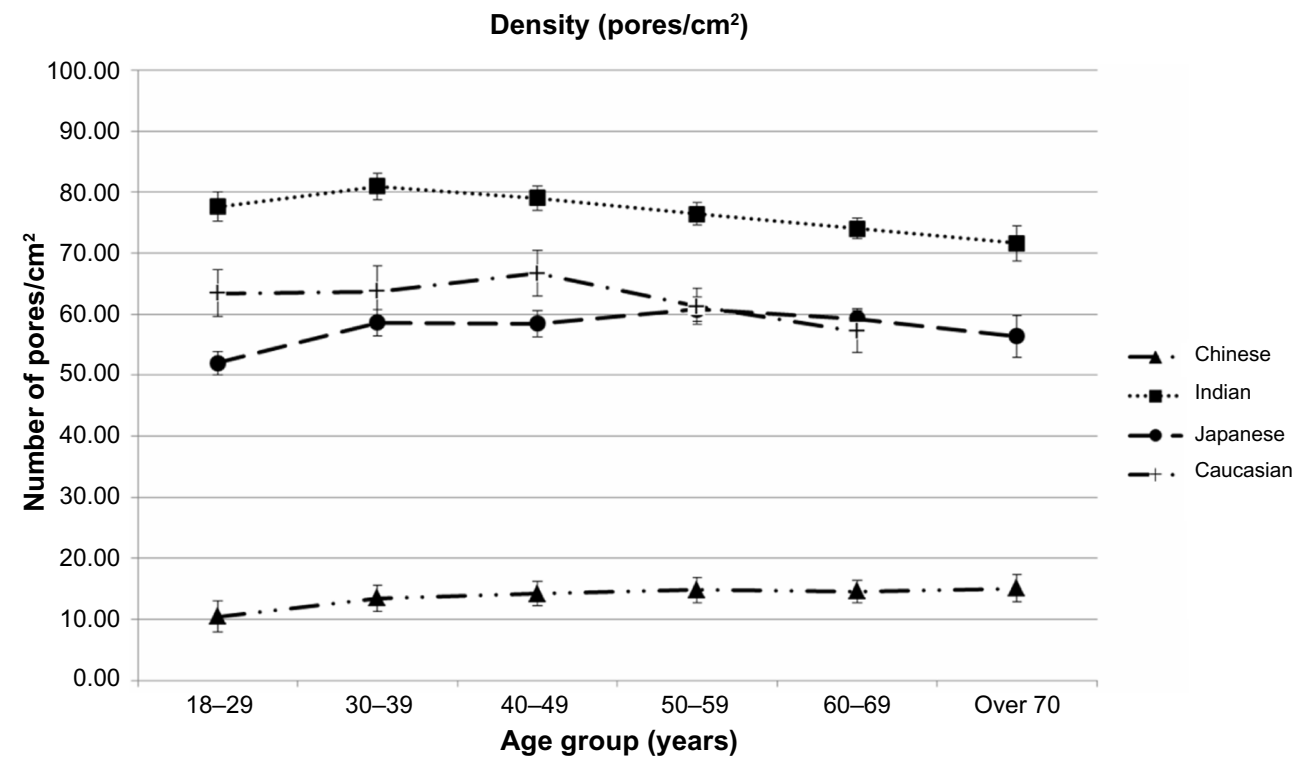

Figure 4 Changes in densities of skin pores with age and ethnic group.

Notes: All 2,585 subjects were included. Data expressed as mean values $\pm 95 \%$ confidence intervals in each age class among ethnic groups. 


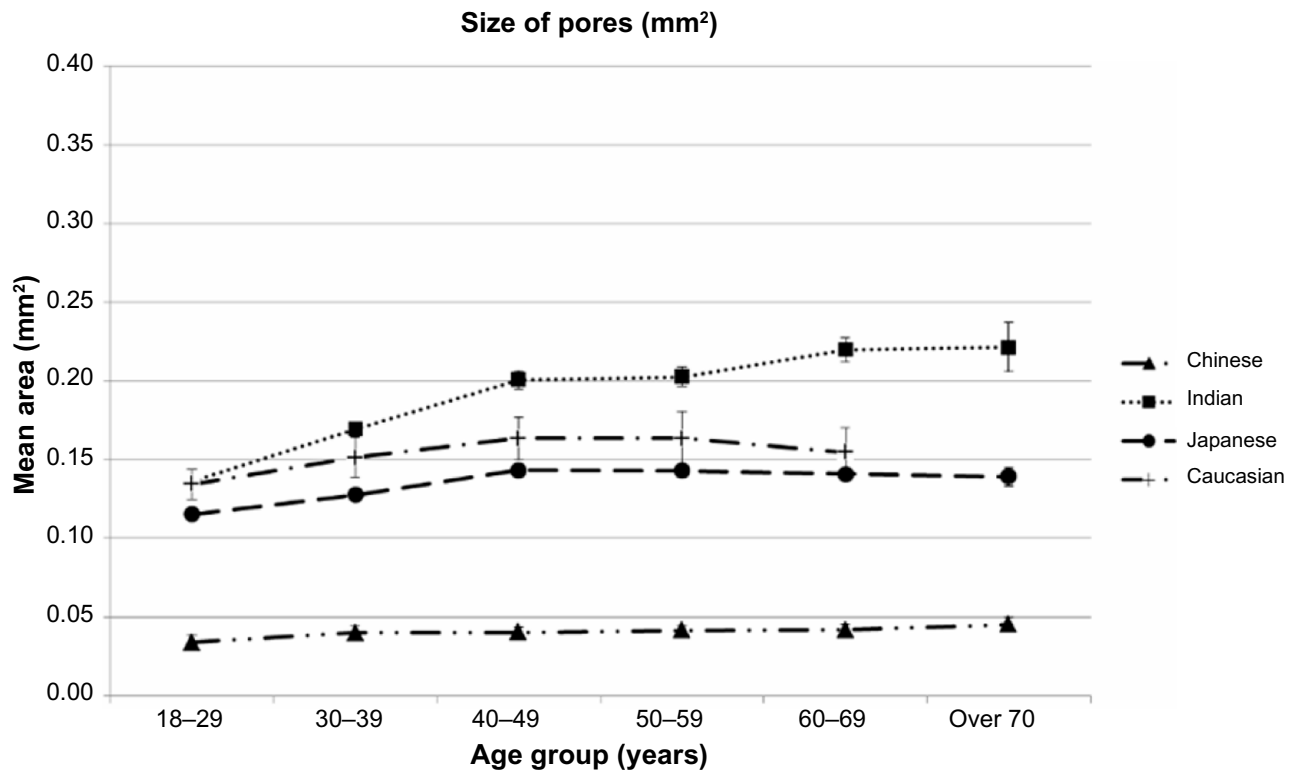

Figure 5 Changes in sizes of skin pores with age and ethnic group.

Notes: All 2,585 subject were included. Data expressed as mean values $\pm 95 \%$ confidence intervals in each age class among ethnic groups.

\section{Effects of ethnicity and age upon the relative area covered by SP}

Figure 6 illustrates the relative part of the surface of the cheeks as a percentage actually covered by SP, with all 2,585 subjects included. Integrating the two previous parameters (densities and sizes) and their respective variances logically amplifies variability among ethnicities, leading to a wide variation in the relative areas, ranging from $1 \%$ to $16 \%$, an approximate factor of 16 .
Apart from among Indian women, between 18 years and 40 years, age appears to nonsignificantly impact such criteria in other ethnic groups.

\section{Focus upon SP among subjects having enlarged SP}

As mentioned above, a special focus was placed on 960 women from five different ethnicities, all self-claiming to have enlarged SP (SP+), and 30\% of subjects with the larg-

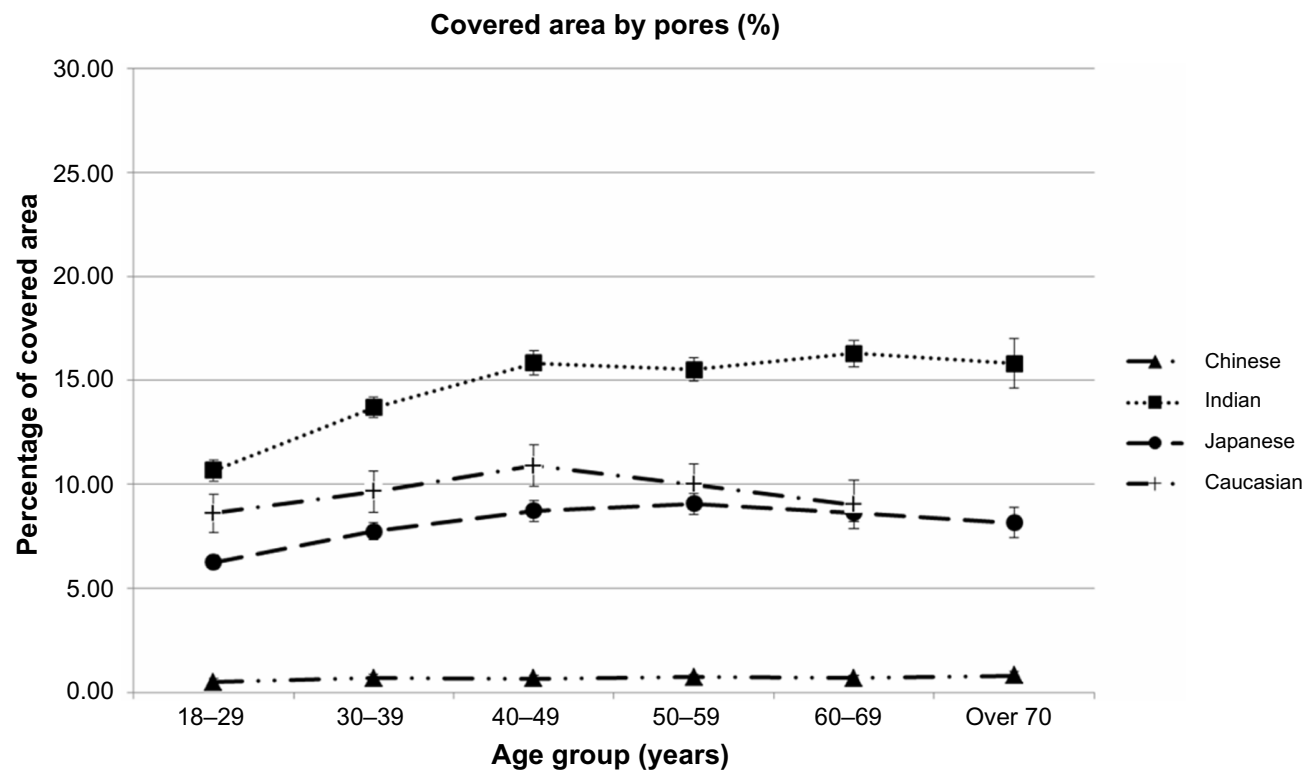

Figure 6 Changes in the relative skin surface covered by skin pores (as a percentage of total skin) with age and ethnicity. Notes: All 2,585 subject included. Data expressed as mean values $\pm 95 \%$ confidence intervals in each age class among ethnic groups. 
est SP in each age class from the studied population $(2,585$ subjects) were gathered, along with an additional group of 120 Brazilian women.

Table 3 gathers the different densities $\left(\mathrm{N} / \mathrm{cm}^{2}\right)$ of $\mathrm{SP}+$ on cheeks of the selected subgroups ( 960 subjects) according to ethnicity and age. The comparison of Table 3 with Figure 4 shows that values logically increase in all cases. For example, the passage from $\mathrm{SP}$ to $\mathrm{SP}+$ induced a change in $10 / \mathrm{cm}^{2}(\mathrm{SP})$ to $21 / \mathrm{cm}^{2}(\mathrm{SP}+$ ). Again, highly variable densities of $\mathrm{SP}+$ were found between ethnic groups, ranging approximately from 20 to $90 / \mathrm{cm}^{2}$, and these values plateaued with an increase in age. Chinese subjects were significantly differentiated from the four other ethnic groups, with much lower values of $\mathrm{SP}+/ \mathrm{cm}^{2}$. Age appeared to have a nonsignificant impact in most ethnic groups on $\mathrm{SP}+/ \mathrm{cm}^{2}$ and led to a significant decrease among Brazilian women.

\section{Effects of ethnicity and age upon size of SP+}

Table 4 gathers the respective average sizes of SP+ according to ethnicity and age in the subgroup of 960 women. Again, a clear ethnic influence can be observed. The youngest age class (18-29 years old) presented a significantly lower size in comparison to all other age groups in every ethnic group. Brazilian and Indian subjects showed an almost regular and significant increase in the size of $\mathrm{SP}+$ with increasing age. Inversely, age did not greatly affect the values in the three other ethnic groups (Caucasian, Japanese, Chinese), which plateaued with age, over 50. According to ethnicity, sizes of SP+ appeared highly variable, ie, ranging approximately from 0.06 to $0.37 \mathrm{~mm}^{2}$ on average, a sixfold increase with regard to extreme values, as compared to the $0.03-0.22$ $\mathrm{mm}^{2}$ range previously mentioned in the global population (2,585 subjects).

Ethnicity comparison, regardless of age, presented significantly different values except for between Caucasian and Indian women from 18 to 39 years of age.

\section{Relative skin surface covered by SP+ according to ethnicity and age}

Table 5 illustrates differences among ethnic groups where the extreme ranges of skin surface covered by $\mathrm{SP}+$ extend from 16-fold in the global population to approximately 19-fold in the subgroup of 960 subjects $(1.2 \%-22.8 \%)$. Age appears to be a rather poor influence on this parameter, apart from in Indian women who showed a significant increase from early ages and plateaued later; Brazilian women were shown to have a rather stable percentage of skin covered by $\mathrm{SP}+$ with age (as shown in Tables 3 and 4).

Ethnicity comparison, regardless of age, presented significantly different values except for between Caucasian and Japanese women from 18 to 69 years of age.

\section{Discussion}

The present study was initially aimed at establishing the global prevalence of SP among ethnic groups and its relation to age. The study confirms the reality of SP as anatomical entities on the cheeks of all women from different ethnic groups. The protocol used in the present work recruited all subjects with rather few exclusion criteria, ie, to observe the common people, leaving aside possible impacts of daily habits such as drug use, food intake, cosmetics use, etc. From a technical viewpoint, the dimensions of the Dermascore $^{\circledR}$ do not allow for easy application to other facial sites such as nose or chin, where SP are indeed present. The cheeks were therefore given privilege in the present work, which is also relevant regarding (internal) studies performed with eye tracker technology or statistical modeling describing the importance of this facial area in full-face perception by woman herself or other people. Common to all cheeks, SP show highly variable sizes and densities according to ethnicity, in rather close agreement with previous findings. ${ }^{13}$ The $250 \mu \mathrm{m}$ threshold that was used allows for detection (assuming a circular shape of pores) of pore sizes equal to or larger than $0.05 \mathrm{~mm}^{2}$, with smaller sizes being barely visible by the experts or subjects.

Table 3 Variable density of enlarged skin pores with ethnicity and age

\begin{tabular}{|c|c|c|c|c|c|c|}
\hline Age group (years) & $18-29$ & $30-39$ & $40-49$ & 50-59 & $60-69$ & Over 70 \\
\hline Brazilian & $91.26 \pm 14.37$ & $81.75 \pm 16.79$ & $68.7 \pm 12.04$ & $61.73 \pm 7.95$ & & \\
\hline Caucasian & $63.96 \pm 11.72$ & $62.22 \pm 11.80$ & $64.49 \pm 8.53$ & $59.93 \pm 7.76$ & $61.13 \pm 8.04$ & \\
\hline Chinese & $21.13 \pm 9.49$ & $23.24 \pm 7.48$ & $20.77 \pm 6.55$ & $24.5 I \pm 6.67$ & $20.20 \pm 6.82$ & $21.66 \pm 8.59$ \\
\hline Indian & $82.68 \pm 15.42$ & $79.91 \pm 12.24$ & $77.12 \pm 9.89$ & $77.53 \pm 10.88$ & $73.56 \pm 7.51$ & $71.19 \pm 9.88$ \\
\hline Japanese & $67.41 \pm 13.95$ & $69.64 \pm 12.10$ & $69.78 \pm|2.7|$ & $72.44 \pm 12.89$ & $69.75 \pm 10.22$ & $68.83 \pm 9.01$ \\
\hline
\end{tabular}

Notes: Data expressed as mean \pm standard deviation in pores $/ \mathrm{cm}^{2}$. Fair Grey tone background (whatever contiguous cell) means significant differences compare to all other age clusters, within a same ethnic group. Same grey tone (only for medium and dark) for contiguous cells illustrates non-significantly different values through age clusters, within same ethnic group. A group of contiguous cells with same grey tones (medium and dark) are significantly different compare to all other age clusters, within same ethnic group. 
Table 4 Variable areas of enlarged skin pores according to ethnics and age

\begin{tabular}{lllllll}
\hline Age group (years) & $\mathbf{1 8 - 2 9}$ & $\mathbf{3 0 - 3 9}$ & $\mathbf{4 0 - 4 9}$ & $\mathbf{5 0 - 5 9}$ & $\mathbf{6 0 - 6 9}$ & $\mathbf{0 v e r ~ 7 0}$ \\
\hline Brazilian & $0.23 \pm 0.06$ & $0.29 \pm 0.08$ & $0.33 \pm 0.07$ & $0.37 \pm 0.00$ & & \\
Caucasian & $0.16 \pm 0.02$ & $0.20 \pm 0.03$ & $0.21 \pm 0.03$ & $0.22 \pm 0.06$ & $0.21 \pm 0.03$ & \\
Chinese & $0.06 \pm 0.01$ & $0.06 \pm 0.01$ & $0.06 \pm 0.01$ & $0.06 \pm 0.01$ & $0.06 \pm 0.01$ & $0.06 \pm 0.01$ \\
Indian & $0.17 \pm 0.02$ & $0.21 \pm 0.02$ & $0.25 \pm 0.02$ & $0.25 \pm 0.03$ & $0.28 \pm 0.03$ & $0.29 \pm 0.03$ \\
Japanese & $0.14 \pm 0.01$ & $0.16 \pm 0.02$ & $0.19 \pm 0.03$ & $0.18 \pm 0.02$ & $0.18 \pm 0.02$ & $0.17 \pm 0.02$ \\
\hline
\end{tabular}

Notes: Data expressed as mean \pm standard deviation in $\mathrm{mm}^{2}$. Fair Grey tone background (whatever contiguous cell) means significant differences compare to all other age clusters, within a same ethnic group. Same grey tone (only for medium and dark) for contiguous cells illustrates non-significantly different values through age clusters, within same ethnic group. A group of contiguous cells with same grey tones (medium and dark) are significantly different compare to all other age clusters, within same ethnic group.

Among the 2,585 studied volunteers, the average values found in this study globally ranged from 0.05 to $0.37 \mathrm{~mm}^{2}$, with high differences among ethnic groups, where Chinese and Japanese women present a clear and significant lower amplitude of variations $\left(0.06-0.18 \mathrm{~mm}^{2}\right)$. These values are in agreement with previous findings despite a different technical approach. ${ }^{3}$ With regard to the impact of sex, the present work only focused on women since SP are a frequent source of complaints among women and a common focus of their wishes to improve their appearance. In addition, a previous work ${ }^{8}$ outlined the lower prevalence of SP in men, despite higher sebum production in men. ${ }^{2,8}$ Further works are nevertheless needed to confirm such difference with sex among the same ethnic groups.

As compared to ethnic influence, age seems to have less affect on SP or, at least, age differently impacts ethnic groups, as observed in the Chinese, French, and Japanese ethnic groups, where both densities and sizes grossly plateaued from approximately 40 years or age. Conversely, Indian and Brazilian women present an obvious trend toward larger pore sizes with increased age. A third case was found in Chinese women who were clearly distinct from other ethnic groups, showing the lowest and the most stable numbers of SP with increasing age.

The slight increase in both size and density, from 18 to 40 years of age, seems a common trend in all ethnic groups. This logically raises questions about numbers and amplitudes of SP at younger ages (0-18 years), where observations on prepubertal children (7-10 years) are crucially lacking.
A total absence of SP among this age population, ethnics including all ethnic groups, would suggest an onset strictly linked to puberty-induced changes in both skin functions and structure.

Interestingly, the subgroups of subjects who self-claimed to have SP+ represented about one-third of the total subjects, a common figure in the five ethnic groups studied. The subjects in this subgroup indeed showed increased values of the three criteria (size, density, percentage of covered skin surface), at comparable ages and ethnicities as compared to the general population, confirming their own visual assessments. Again, Indian and Brazilian women were observed to have increases in SP size of much larger amplitudes than those observed in Asian and Caucasian subjects. Among these SP+ subgroups, Brazilian women strikingly showed much larger pore sizes as well as a strong and almost linear trend of increased SP size with increased with age. Densities appeared little-affected in all subgroups, showing rather stable intrinsic values with age, or even declining with age in Brazilian and Indian women.

To summarize, pore sizes vary much more with ethnicity than with age. With regard to women claiming to have $\mathrm{SP}+$, the most extreme average values of which there was significant difference between ethnicities was between Chinese $\left(0.05 \mathrm{~mm}^{2}\right)$ and Brazilian $\left(0.37 \mathrm{~mm}^{2}\right)$ women, with a sevenfold variation in size. These figures are visually amplified since up to $23 \%$ of the total skin surface of the cheeks is composed of SP, an amount high enough to be self-observed. In other words, these results suggest that, for an individual's self-perception, larger pores (although less frequent) bring a stronger subjective resent

Table 5 Percentages of skin surface covered by enlarged skin pores with age for five ethnic groups

\begin{tabular}{lllllll}
\hline Age group (years) & $\mathbf{1 8 - 2 9}$ & $\mathbf{3 0 - 3 9}$ & $\mathbf{4 0 - 4 9}$ & $\mathbf{5 0 - 5 9}$ & $\mathbf{6 0 - 6 9}$ & $\mathbf{O v e r} \mathbf{7 0}$ \\
\hline Brazilian & $20.74 \pm 2.77$ & $22.8 I \pm 2.34$ & $22.47 \pm 3.41$ & $22.84 \pm 2.67$ & & $12.96 \pm 2.27$ \\
Caucasian & $10.58 \pm 2.89$ & $12.11 \pm 2.61$ & $13.27 \pm 2.01$ & $12.80 \pm 2.35$ & $1.19 \pm 0.60$ & $1.48 \pm 0.92$ \\
Chinese & $1.23 \pm 0.68$ & $1.46 \pm 0.78$ & $1.21 \pm 0.49$ & $1.48 \pm 0.63$ & $20.33 \pm 2.35$ & $20.39 \pm 2.65$ \\
Indian & $13.68 \pm 3.14$ & $16.55 \pm 2.91$ & $19.29 \pm 3.17$ & $19.18 \pm 2.50$ & 12.35 \\
Japanese & $9.58 \pm 2.46$ & $11.02 \pm 2.72$ & $13.02 \pm 2.97$ & $13.4 \pm 3.18$ & $12.27 \pm 2.54$ & $11.82 \pm 2.32$ \\
\hline
\end{tabular}

Notes: Data expressed as mean \pm standard deviation in\%. Fair Grey tone background (whatever contiguous cell) means significant differences compare to all other age clusters, within a same ethnic group. Same grey tone (only for medium and dark) for contiguous cells illustrates non-significantly different values through age clusters, within same ethnic group. A group of contiguous cells with same grey tones (medium and dark) are significantly different compare to all other age clusters, within same ethnic group. 
that is possibly levered by cultural and traditional aesthetic standards. Such would attribute variable aesthetical impact (self-esteem) to a benign clinical feature, regardless of its amplitude, Japanese women being likely an illustrative case. Regardless, these results clearly contradict the common belief in Japan, Europe, and particularly in the People's Republic of China that, with age, SP become more numerous and/or larger. From a cosmetic viewpoint, SP and their high prevalence in some ethnic groups fully justify an efficient camouflaging strategy.

The origin of SP remains puzzling, since hardly integrating clinical pictures classified by textbooks of Dermatology. Their benign nature, lack of a specific pigmentation, privileged facial occurrence (cheeks, nose, chin) in both sexes, ${ }^{1,5,8,13}$ the fact that they are unrelated to evident skin afflictions, etc, mean that the clinical concerns of SP are limited to concerns of aesthetics.

Is sun exposure another factor that significantly increases SP, ${ }^{14}$ making SP another feature of photo-altered skin, with the face being the skin region drawing the most concern? In fact, topical applications of Tretinoin ${ }^{\mathrm{TM}}$, Vitamin C, or AHAs have significantly mitigating impacts upon $\mathrm{SP}^{5,15,16}$

In disagreement with some works, ${ }^{8-10}$ we do not particularly support the view that SP primarily originate from a sebaceous dysfunction or an excessive sebum production for the following reasons. First, positive correlations between SP and sebum output do not ipso facto imply causal relations. In contrast, a previous work ${ }^{8}$ described a lower presence of SP among men, as compared to women, despite their higher sebum production. ${ }^{8,17}$ However, it is likely that a sebuminduced shine could make SP more visible and ostensibly enlarged to the naked eye, and the same is true of the evolution of emulsion sebum/sweat. Second, sizes and densities of SP appear to be overall rather constant along the lifespan, whereas the sebaceous function, in all ethnic groups, regularly declines after 40 years of age. ${ }^{17-19}$ Third, previous internal studies (Flament, unpublished data, 2012) have shown that sebum production in Indian subjects, despite their large and numerous SP, has the lowest values among all ethnic groups. Fourth, there is conflicting evidences vis à vis past acne - which is closely linked to the sebaceous function - and its influence upon size and numbers of SP. ${ }^{10,20}$

Biophysical measurements (sebum output, elastic properties $)^{10}$ are difficult to interpret due to unavoidable bias. On one hand, SP occupy at most $25 \%$ of the total surface of skin, a value that obviously "dilutes" a possible specific signal upon the physical properties of the skin. On the other hand, comparison with a control, ie, skin free from SP, is a difficult objective since SP affects both cheeks in all subjects, at comparable ages, presenting a symmetric pattern.

The structural features of SP, as small convex cuvettes conferring a "golf-ball"-like aspect to the skin, are highly suggestive of early-induced epidermal and dermal impairments, as evidenced by deeper observations through noninvasive confocal laser microscopy. ${ }^{7}$ This noninvasive confocal laser microscopy showed structural changes in dermal papilla and an isotropic dermal fiber structure that underlies SP. These observations are, we believe, highly relevant as they are in agreement with associations to functional criteria through growth factors (IGF-1) $)^{21}$ and hormonal balance that strongly controls epidermal, dermal, and skin appendages, ${ }^{22,23}$ including sebaceous function, which are all greatly affected by puberty.

The reported effect of botulinum toxin upon $\mathrm{SP}^{9}$ is, we believe, more linked to its potent effect upon dermal and muscular compartments than upon the sebaceous function, as previously suggested.

However, to what extent SP may be enlarged in size and number by sun exposure or lifestyle (which are possible additional contributors to photo-damaged skin) remains an open issue that clearly calls for further works, which should not discount a participating role of sebaceous function. SP nevertheless remain puzzling; the hope is that future works will result in greater understanding. ${ }^{1}$

\section{Acknowledgments}

We deeply want to thank Mr Didier Saint-Leger, Mrs Shweta Purandare, Mrs Virginie Hourblin, Mrs Xiaohui Long, Mr Loïc Colomb, Mr Stephane Diridollou, Mrs Odette Jammayrac, Mrs Veronique Burnier, Mr Stephane Ortiz, and Mr Bertrand Piot, not only for their great help in the completion of this paper and global study, but also for their strong support and enthusiasm.

\section{Disclosure}

The authors report no conflicts of interest in this work.

\section{References}

1. Uhoda E, Piérard-Franchimont C, Petit L, Piérard GE. The conundrum of skin pores in dermocosmetology. Dermatology. 2005;210(1):3-7.

2. Plewig G, Kligman AM. Acne and Rosacea. 3rd edition. Berlin, Heidelberg: Springer; 2000.

3. Holyoke JB, Lobitz WC Jr. Histologic variations in the structure of human eccrine sweat glands. J Invest Dermatol. 1952;18:147-167.

4. Jo HY, Yu DS, Oh CH. Quantitative research on skin pore widening using a stereoimage optical topometer and Sebutape. Skin Res Technol. 2007;13(2):162-168.

5. Kakudo N, Kushida S, Tanaka N, Minakata T, Suzuki K, Kusumoto K. A novel method to measure conspicuous facial pores using computer analysis of digital-camera-captured images: the effect of glycolic acid chemical peeling. Skin Res Technol. 2011;17(4):427-433. 
6. Saedi N, Petrell K, Arndt K, Dover J. Evaluating facial pores and skin texture after low-energy nonablative fractional 1440-nm laser treatments. J Am Acad Dermatol. 2013;68(1):113-118.

7. Mizukoshi K, Takahashi K. Analysis of the skin surface and inner structure around pores on the face. Skin Res Technol. 2014;20(1):23-29.

8. Roh M, Han M, Kim D, Chung K. Sebum ouput as a factor contributing to the size of facial pores. Br J Dermatol. 2006;155(5):890-894.

9. Shah AR. Use of intradermal botulinum toxin to reduce sebum production and facial pore size. J Drugs Dermatol. 2008;7(9):847-850.

10. Kim BY, Choi JW, Park KC, Youn SW. Sebum, acne, skin elasticity and gender difference - which is the major influencing factor for facial pores? Skin Res Technol. 2013;19(1):e45-e53.

11. François G, Maudet A, McDaniel D, Giron F, Bazin R. Quantification of facial pores using image analysis. Cosmetic Dermatology. 2009;22(9): 457-463.

12. Bazin R, Flament F. Skin Aging Atlas. Volume 2, Asian Type. Paris: Editions Med'Com; 2010.

13. Hillebrand GG, Levine JM, Miyamoto K. The age-dependent changes in skin condition in ethnic populations from around the world. In: Berardesca E, Lévêque JL, Maibach HI, editors. Ethnic Skin and Hair (Dermatology: Clinical and Basic Science Series/28). New-York, London: Informa; 2007:105-122.

14. Flament F, Bazin R, Qiu H, et al. Solar exposure(s) and facial clinical signs of aging in Chinese women: Impacts upon age perception. Clin Cosmet Investig Dermatol. In Press.

15. Lowe PM, Woods J, Lewis A, Davies A, Cooper AJ. Topical tretinoin improves the appearance of photo damaged skin. Australas J Dermatol. 1994;35(1):1-9.
16. Xu TH, Chen JZ, Li YH, et al. Split-face study of topical $23.8 \%$ L-ascorbic acid serum in treating photo-aged skin. J Drugs Dermatol. 2012;11(1):51-56.

17. Jacobsen E, Billings JK, Frantz RA, Kinney CK, Stewart ME, Downing DT. Age-related changes in sebaceous wax ester secretion rates in men and women. J Invest Dermatol. 1985;85(5):483-485.

18. Makrantonaki E, Adjaye J, Herwig R, et al. Age-specific hormonal decline is accompanied by transcriptional changes in human sebocytes in vitro. Aging Cell. 2006;5(4):331-344.

19. Makrantonaki E, Zouboulis CC; German National Genome Research Network 2. The skin as a mirror of the aging process in the human organism - state of the art and results of the aging research in the German National Genome Research Network 2 (NGFN-2). Exp Gerontol. 2007;42(9):879-886.

20. Perkins AC, Maglione J, Hillebrand GG, Miyamoto K, Kimball AB. Acne vulgaris in women: prevalence across the life span. $J$ Womens Health (Larchmt). 2012;21(2):223-230.

21. Sugiyama-Nakagiri Y, Naoe A, Ohuchi A, Kitahara T. Serum levels of IGF-1 are related to human skin characteristics including the conspicuousness of facial pores. Int J Cosmet Sci. 2011;33(2):144-149.

22. Suigiyama-Nakagiri Y, Sugata K, Hachiya A, Osanai O, Ohuchi A, Kitahara T. Ethnic differences in the structural properties of facial skin. J Dermatol Sci. 2009;53:135-139.

23. Kanda N, Watanabe $\mathrm{S}$. Regulatory roles of sex hormones in cutaneous biology and immunology. J Dermatol Sci. 2005;38(1):1-7.
Clinical, Cosmetic and Investigational Dermatology

\section{Publish your work in this journal}

Clinical, Cosmetic and Investigational Dermatology is an international, peer-reviewed, open access, online journal that focuses on the latest clinical and experimental research in all aspects of skin disease and cosmetic interventions. All areas of dermatology will be covered; contributions will be welcomed from all clinicians and

\section{Dovepress}

basic science researchers globally. This journal is indexed on CAS. The manuscript management system is completely online and includes a very quick and fair peer-review system, which is all easy to use. Visit http://www.dovepress.com/testimonials.php to read real quotes from published authors. 